\title{
Hyper-generalized-gradient functionals constructed from the Lieb-Oxford bound: Implementation via local hybrids and thermochemical assessment
}

\author{
Robin Haunschild, ${ }^{1}$ Mariana M. Odashima, ${ }^{2, a)}$ Gustavo E. Scuseria, ${ }^{1,3}$ John P. Perdew, ${ }^{4}$ \\ and K. Capelle ${ }^{5}$ \\ ${ }^{1}$ Department of Chemistry, Rice University, Houston, Texas 77005, USA \\ ${ }^{2}$ Max-Planck Institut für Mikrostrukturphysik, Weinberg 2, D-06120 Halle, Germany \\ ${ }^{3}$ Department of Physics and Astronomy, Rice University, Houston, Texas 77005, USA and Chemistry \\ Department, Faculty of Science, King Abdulaziz University, Jeddah 21589, Saudi Arabia \\ ${ }^{4}$ Department of Physics and Quantum Theory Group, Tulane University, New Orleans, Louisiana 70118, USA \\ ${ }^{5}$ Centro de Ciências Naturais e Humanas, Universidade Federal do ABC - Santo André, \\ 09210-170 São Paulo, Brazil
}

(Received 20 January 2012; accepted 23 April 2012; published online 9 May 2012)

\begin{abstract}
In 2009 Odashima and Capelle (OC) showed a way to design a correlation-only density functional that satisfies a Lieb-Oxford bound on the correlation energy, without empirical parameters and even without additional theoretical parameters. However, they were only able to test a size-inconsistent version of it that employs total energies. Here, we show that their alternative size-consistent form that employs energy densities, when combined with exact or semilocal exchange, is a local hybrid (lh) functional. We test several variants of this nonempirical OC-lh functional on standard molecular test sets. Although no variant yields enthalpies of formation with the accuracy of the semilocal TaoPerdew-Staroverov-Scuseria (TPSS) exchange-correlation, OC-lh correlation with exact exchange yields rather accurate energy barriers for chemical reactions. Our purpose here is not to advocate for a new density functional, but to explore a previously published idea. We also discuss the importance of near-self-consistency for fully nonlocal functionals. () 2012 American Institute of Physics. [http://dx.doi.org/10.1063/1.4712017]
\end{abstract}

\section{INTRODUCTION}

The key to the success of computational quantum chemistry and solid-state physics is the accurate description of beyond mean-field many-body interactions, which in KohnSham density-functional theory (DFT) (Refs. 1-3) reside in the exchange-correlation energy functional $\left(E_{x c}\right)$. Although today's approximate density functionals often provide a good description of many properties of atoms, molecules, and solids, there is still significant demand for new density functionals and for new approaches to their construction. Especially, better non-empirical density functional approximations (DFAs) are needed, as many well-performing DFAs contain fitted parameters. In this paper, we investigate a class of nonlocal functionals based on controlled interpolation between upper and lower bounds of $E_{x c}$.

An obvious upper bound on the exchange-correlation energy is zero, as a consequence of the variational principle and the definition of the exchange energy. The existence of a lower bound is much less trivial, but was established by Lieb ${ }^{4}$ and Lieb and Oxford ${ }^{5}$ in the context of work on the stability of nonrelativistic matter. The form of this bound adopted in DFT is ${ }^{6,7}$$$
E_{x} \geq E_{x c}=E_{x}+E_{c} \geq-C \int d^{3} r n^{4 / 3}(\mathbf{r}) \equiv \lambda E_{x}^{\mathrm{LDA}}[n],
$$

where $C$ and $\lambda=1.354 C$ are universal constants and $E_{x}^{\mathrm{LDA}}$ is the local-density approximation (LDA) (Ref. 8) to the ex-

\footnotetext{
a) odashima@mpi-halle.mpg.de.
}

change energy. This bound, known as the Lieb-Oxford (LO) bound ${ }^{5}$ is a property of nonrelativistic quantum mechanics applied to many-electron systems, and thus holds across chemistry and physics, regardless of whether one employs wave-function-based methods or density-functional methods.

The estimate for the maximum value of the prefactor $C$ provided by Lieb and Oxford is $C_{\mathrm{LO}}=1.68$ (corresponding to $\lambda_{\mathrm{LO}}=2.27$ ). Later, Chan and Handy ${ }^{9}$ derived a slightly tighter prefactor, $C \leq 1.64$. Recently it was proposed, based on empirical analysis of a wide range of real and model systems and backed up by physical arguments, ${ }^{10-13}$ that the tightest possible value should be $\lambda=1.9555$ (corresponding to $C$ $=1.44) .{ }^{10}$ The $\mathrm{LO}$ value has been employed as a constraint in the construction of various density functionals for the exchange energy, such as the Perdew-Burke-Ernzerhof (PBE) (Ref. 14) and the Tao-Perdew-Staroverov-Scuseria (TPSS). ${ }^{15}$ A reduction of the value of $\lambda$ in PBE can by itself benefit bond lengths of small molecules ${ }^{16}$ and equilibrium lattice constants. ${ }^{17}$ Other extensions of the PBE functional form that effectively or explicitly reduce the value of the LO constraint, achieved improved molecular atomization energies, ${ }^{18}$ and very good lattice constants. ${ }^{19}$

A useful, although not all encompassing, classification of density functionals is afforded by Jacob's ladder, ${ }^{20,21}$ according to which functionals are assigned to different rungs of a ladder depending on the local ingredients they use. Thus, on rung one we have the local-(spin)-density approximation (L(S)DA), while rung two comprises gradient-dependent approximations, of which the generalized-gradient approximations (GGAs) are the most popular ones. ${ }^{21}$ On the third rung 
we have the meta-GGA (MGGA) functionals, making use of the kinetic-energy density or equivalent quantities as local ingredients, while rung four is reserved for hyper-GGA (HGGA) functionals, employing the exchange-energy density as a local ingredient of the correlation energy. In more recent usage, rung four is extended to include any functional with any exact-exchange ingredient, therefore comprising hybrid functionals, which mix exact and approximate exchange, but also other forms of functional constructions. In the present paper we use the expressions "hybrid functional" and "hyper GGA" interchangeably, because for the class of functionals investigated here one can always be rewritten in the form of the other.

A key step in the construction of GGA, MGGA, and HGGA functionals is the choice of the algebraic form of the model for the corresponding energy density. In many common functionals, this step is guided by the requirement to satisfy exact constraints, such as the Lieb-Oxford bound. PBE GGA (Ref. 14) and TPSS MGGA, ${ }^{15}$ for example, employ the LO value of the prefactor, which is used to fix the value of a parameter $(\kappa)$ appearing in the chosen algebraic form of the exchange-energy density. ${ }^{14,15}$ By contrast, here we are concerned with a recently proposed family of correlation-energy functionals ${ }^{22}$ which employ the LO bound in a very different way: instead of fixing a parameter in a chosen form for the functional, in this family of functionals the bound is used for constraining the form of the functional itself. Remarkably, the resulting functionals, not constructed by systematic inclusion of local ingredients on top of L(S)DA, still find their place on Jacob's ladder, where they naturally appear as hyper-GGAs. ${ }^{22}$ Moreover, as we detail below, they can also be cast as a family of local or global hybrid functionals.

While functionals on the first three rungs of the ladder have been constructed without empirical fitting, almost all fourth-rung functionals have at least some such fitting. Thus, a special interest attaches to the family of functionals of Ref. 22, which are nonempirical.

The present paper first describes, in Sec. II, the connection of this family of LO-based functionals to hybrid functionals. One representative of this family was implemented in a development version of the GAUSSIAN code ${ }^{23}$ and tested on heats of formation and barrier heights of gas-phase chemical reactions, against standard nonempirical LSDA, GGA, MGGA, and hybrid functionals. Results are presented and analyzed in Sec. III. Finally, Sec. IV contains our conclusions and a list of open questions.

\section{HYPER-GGA FUNCTIONALS CONSTRUCTED FROM THE LIEB-OXFORD BOUND}

\section{A. Construction employing total energies}

In 2009 Odashima and Capelle (OC) (Ref. 22) showed that, up to a prefactor that is itself a functional of the density, the universal correlation functional of DFT can be cast as a difference of two exchange functionals,

$$
E_{c}[n]=\beta[n]\left(\lambda E_{x}^{\mathrm{LDA}}[n]-E_{x}[n]\right),
$$

where $E_{x}[n]$ is the exact exchange energy, $E_{x}^{\mathrm{LDA}}[n]$ is its local approximation $^{8,24,25}$ and $\lambda$ is the Lieb-Oxford constant. From here on, most approximations to $E_{x}[n]$ and $E_{c}[n]$ can be and in our work are spin-density functionals, but $E_{x}^{\mathrm{LDA}}[n]$ from Eq. (2) remains a functional of the total density $n(\mathbf{r})=n_{\uparrow}(\mathbf{r})$ $+n_{\downarrow}(\mathbf{r})$.

This representation of $E_{c}[n]$ would be trivial if the functional $\beta[n]$ was arbitrary, since it would then just amount to replacing one unknown functional, $E_{c}[n]$, by another, $\beta[n]$. However, by combining the variational theorem with the Lieb-Oxford bound in formulating the upper and lower limits of the interpolation, the OC-construction guarantees that while $E_{c}[n]$ can take on values in the range of $-\infty$ to 0 , the prefactor $\beta[n]$ can vary only from 0 to 1 . This reduced variability makes Eq. (2) particularly interesting from the point of view of development of approximations to $E_{c}[n]$.

OC further showed ${ }^{22}$ that, by imposing the correct behaviour for the uniform electron gas and the correct recovery of the low-order gradient expansion for weakly spatially varying systems, simple approximations for $\beta[n]$ can be developed that obey the same or similar sets of exact conditions as standard GGA and post-GGA functionals, and are also numerically competitive with these. The basic form of the resulting family of functionals is

$$
E_{c}^{\mathrm{OC}, \mathrm{HGGA}}[n]=\frac{\tilde{E}_{c}[n]}{\lambda E_{x}^{\mathrm{LDA}}[n]-\tilde{E}_{x}[n]}\left(\lambda E_{x}^{\mathrm{LDA}}[n]-E_{x}[n]\right),
$$

where $\tilde{E}_{c}[n]$ and $\tilde{E}_{x}[n]$ are any expressions that on constant densities reduce to the correlation and exchange energy of the uniform electron gas, respectively, and on weakly varying densities reduce to the respective gradient expansions. The choice of these expressions is, of course, not unique. In fact, the functional $\tilde{E}$ used need not even be stand-alone exchange and correlation functionals, as all that is required of them is to reproduce the correct limits on constant and weakly varying densities. Moreover, to provide the cleanest possible test of the effect of a possible reduction of $\lambda$, the functional used for constructing the prefactor should ideally not itself have an explicit $\lambda$-dependence, but should obey the LO bound. These requirements exclude several widely used functionals, including PBE, and single out the Perdew-Wang 1991 GGA (PW91) (Ref. 26) for exchange and for correlation as a suitable candidate for $\tilde{E}_{c}[n]$ and $\tilde{E}_{x}[n]$. The resulting functional reads ${ }^{22}$

$$
E_{c}^{\mathrm{OC}, \mathrm{HGGA}-1}[n]=\frac{E_{c}^{\mathrm{PW} 91}[n]}{\lambda E_{x}^{\mathrm{LDA}}[n]-E_{x}^{\mathrm{PW} 91}[n]}\left(\lambda E_{x}^{\mathrm{LDA}}[n]-E_{x}[n]\right) .
$$

When evaluated on PW91 densities, this functional improves on the (already very good) PW91 correlation energies of atoms and small molecules, performing similarly to the Lee-Yang-Parr (LYP) (Ref. 27) correlation, which has three empirical parameters fitted to atomic energies. On the other hand, resulting molecular atomization energies were not competitive. ${ }^{22}$ Part of this failure can be compensated by other choices of approximate functionals in modelling of $\tilde{E}_{c}[n]$ and $\tilde{E}_{x}[n]$. A more fundamental issue, not addressed by such alternative models, is that the functional equation (4) is not sizeconsistent, ${ }^{28}$ as it contains total energies in the denominator. In the original OC work it was already proposed ${ }^{22}$ that this problem could be solved by applying the same construction to 
energy densities instead of integrated energies, but no numerical evaluation of this proposal was made. Along completely different lines the construction to include the exact exchangeenergy density $e_{x}(\mathbf{r})$ as ingredient in $E_{c}[n]$ was introduced by Perdew and collaborators in $1998,{ }^{20,29}$ which lends support to continued construction of HGGA-type functionals by developing approximations to $\beta[n]$.

\section{B. Construction employing energy densities}

In the present paper we implement and test this proposal as well as a couple of other potentially useful modifications of the original construction. First, we replace the PW91 GGA by a higher rung functional, the TPSS meta-GGA, ${ }^{15}$ which imposes additional constraints such as the recovery of the fourth-order gradient expansion of the exchange energy, ${ }^{30}$ and the correct one-electron limit for correlation. Second, we consider both the Lieb-Oxford value $\lambda_{\mathrm{LO}}=2.27$ and the conjectured tighter limit of $\lambda_{\mathrm{OC}}=1.9555,{ }^{10-12}$ as well as a different gauge for the exact energy density, constructed specifically to be compatible with TPSS. ${ }^{31}$ Third, we evaluate the functional on both LSDA and TPSS orbitals, in order to assess the effect of closer approach to self-consistent HGGA orbitals. Finally, we start the OC construction from the local Lieb-Oxford bound, ${ }^{32} e_{x c}(\mathbf{r}) \geq \lambda e_{x}^{\mathrm{LDA}}(\mathbf{r})$, instead of the global one, ${ }^{5}$ in order to recast the $\mathrm{OC}$ construction in terms of energy densities. A local Lieb-Oxford bound has not been proved within any gauge for the exact energy density, but, within a semilocal approximation, the global bound can only be guaranteed for all possible electron densities by satisfaction of the local bound. ${ }^{14,33}$

The fundamental representation of the correlation energy then becomes

$$
e_{c}(\mathbf{r})=\beta[n](\mathbf{r})\left(\lambda e_{x}^{\mathrm{LDA}}(\mathbf{r})-e_{x}(\mathbf{r})\right)
$$

and the resulting correlation energy functional reads

$$
E_{c}^{\mathrm{OC}}[n]=\int d^{3} r \frac{e_{c}^{\mathrm{TPSS}}(\mathbf{r})}{\lambda e_{x}^{\mathrm{LDA}}(\mathbf{r})-e_{x}^{\mathrm{TPSS}}(\mathbf{r})}\left(\lambda e_{x}^{\mathrm{LDA}}(\mathbf{r})-e_{x}(\mathbf{r})\right)
$$

We note that the functional equation (6) is size-consistent but makes use of the local Lieb-Oxford bound, which is not rigorous. In fact, the local bound does not hold when $e_{x}$ is in the conventional gauge. If the local bound holds when $e_{x}$ is in the TPSS gauge, ${ }^{31}$ then Eq. (6) will also satisfy the global Lieb-Oxford bound. On the other hand, the functional Eq. (4) is not size-consistent, but is based on the rigorous global LiebOxford bound. Other available HGGA functionals are typically size-consistent but either also employ the local bound ${ }^{34}$ or do not consider the Lieb-Oxford bound at all, and thus potentially violate both the local and the global form of it. ${ }^{35,36}$ It remains to be explored how size consistency and satisfaction of the global LO bound can be reconciled in the same HGGA functional.

\section{Relation to local hybrids}

Although constructed along completely different lines, there is a tight relation between the HGGAs obtained from the OC construction and hybrid functionals. In fact, to each member of the family of HGGA correlation functionals resulting from the OC construction correspond two hybrid functionals, one obtained from combining HGGA correlation with exact exchange, the other from combining it with approximate exchange. This relation provides a different viewpoint on the structure of hybrid functionals. ${ }^{22}$ It is also very useful in practice for the implementation of these HGGAs, as hybrid functionals are implemented much more widely than HGGA correlation functionals. In the particular case of HGGAs obtained from the local Lieb-Oxford bound, the relation can be made explicit by equating the generic local hybrid ${ }^{29,34,37}$ with the exchange and correlation energy density corresponding to the chosen HGGA,

$$
\begin{aligned}
& a(\mathbf{r}) e_{x}(\mathbf{r})+(1-a(\mathbf{r})) \tilde{e}_{x}(\mathbf{r})+\tilde{e}_{c}(\mathbf{r}) \\
& \quad=\bar{e}_{x}(\mathbf{r})+\frac{\tilde{e}_{c}(\mathbf{r})}{\lambda e_{x}^{\mathrm{LDA}}(\mathbf{r})-\tilde{e}_{x}(\mathbf{r})}\left(\lambda e_{x}^{\mathrm{LDA}}(\mathbf{r})-e_{x}(\mathbf{r})\right),
\end{aligned}
$$

where $a(\mathbf{r})$ is the weight factor of the local hybrid, $e_{x}$ is always the exact exchange-energy density, while both $\bar{e}_{x}$ can be the exact one or an approximation, and $\tilde{e}$ is normally an approximation. If the approximations were replaced by exact functionals, of course, then Eq. (7) would yield the exact $E_{x c}$. Whatever choice is made for $\tilde{e}_{c}$ and $\tilde{e}_{x}$, this equation can be solved for $a(\mathbf{r})$, thus determining the weight factor that must be used in a local hybrid in order to reproduce from it the values obtained from the corresponding HGGA. In the present case, we take TPSS exchange and correlation on the righthand side, and, for convenience, also adopt TPSS exchange for $\tilde{e}_{x}$ on the left-hand side. The result is that the OC HGGA for correlation is equivalent to a local hybrid with weight factor

$$
a(\mathbf{r})=1-\frac{e_{c}^{\mathrm{TPSS}}(\mathbf{r})}{\lambda e_{x}^{\mathrm{LDA}}(\mathbf{r})-e_{x}^{\mathrm{TPSS}}(\mathbf{r})}
$$

when combined with exact exchange, and

$$
a(\mathbf{r})=-\frac{e_{c}^{\mathrm{TPSS}}(\mathbf{r})}{\lambda e_{x}^{\mathrm{LDA}}(\mathbf{r})-e_{x}^{\mathrm{TPSS}}(\mathbf{r})} .
$$

when combined with approximate (TPSS) exchange. These relations imply that OC HGGA, as well as all other members of the OC family of local HGGAs, can be implemented rather straightforwardly in any code featuring local hybrids. In both cases, the TPSS energy densities are functionals of the separate spin up ( $\uparrow)$ and spin down $(\downarrow)$ electron densities, but are not resolved into $\uparrow \uparrow, \uparrow \downarrow$, and $\downarrow \downarrow$ contributions.

It is interesting to remark that these two weight factors, or local mixing functions, lead to quite different local hybrids (lhs). By construction, the energy ratio in the rhs of Eq. (8) is positive, therefore the resulting $a(\mathbf{r})$ is positive and smaller than unity. On the other hand, in Eq. (9) the local mixing function is negative, adding a more significant amount of TPSS exchange in the local hybrid. We will verify how these two local hybrids perform compared to the combination of HartreeFock exchange with TPSS correlation (HFx + TPSS $c$ ) and to pure TPSS. Note that only Eq. (8) and not Eq. (9) has the proper high-density limit $a(\mathbf{r}) \rightarrow 1$ discussed in Ref. 34 .

MGGA and HGGA are orbital-dependent functionals, whose self-consistent evaluation in DFT either requires the 
TABLE I. Mean and mean absolute errors of enthalpies of formation over three standard data sets in $\mathrm{kcal} / \mathrm{mol}$. Standard computational approaches in the first group of lines [HF, LSDA (SVWN5), PBE GGA, and TPSS MGGA] are compared to various combinations of exact (Hartree-Fock) exchange with TPSS correlation (second group), of exact (Hartree-Fock) exchange with OC-lh correlation (third group) and of approximate TPSS exchange with OC-1h correlation. The densities on which the respective functionals have been evaluated are specified within brackets. Where no density is given, the functional has been evaluated self-consistently on its own density. The line corresponding to the overall best functional in each group is set in boldface. Negative mean errors indicate overbinding.

\begin{tabular}{|c|c|c|c|c|c|c|}
\hline & \multicolumn{2}{|c|}{ G2/97 } & \multicolumn{2}{|c|}{ G3-3 } & \multicolumn{2}{|c|}{ G3/99 } \\
\hline & ME & MAE & ME & MAE & ME & MAE \\
\hline $\mathrm{HF}$ & 147.9 & 148.0 & 335.6 & 335.6 & 211.1 & 211.1 \\
\hline LSDA(SVWN5) & -82.8 & 82.8 & -195.3 & 195.3 & -120.6 & 120.6 \\
\hline PBE & -16.1 & 16.9 & -32.6 & 32.6 & -21.6 & 22.2 \\
\hline TPSS & -5.7 & 6.4 & -6.4 & 6.5 & -6.0 & 6.5 \\
\hline$(\mathrm{HF} x+\operatorname{TPSS} c)\left[n^{\mathrm{LSDA}}\right]$ & 33.3 & 34.5 & 48.5 & 48.6 & 38.4 & 39.2 \\
\hline$(\mathrm{HF} x+\operatorname{TPSS} c)\left[n^{\mathrm{TPSS}}\right]$ & 33.5 & 34.7 & 49.2 & 49.2 & 39.6 & 39.6 \\
\hline HF $x+$ TPSS $c$ & 25.2 & 27.7 & 32.6 & 35.4 & 27.7 & 30.3 \\
\hline$(\mathrm{HF} x+$ OC-lhc $)\left[n^{\text {TPSS }}\right]$, conv. gauge & 34.1 & 35.2 & 53.0 & 53.0 & 40.4 & 41.2 \\
\hline$(\mathrm{HF} x+\mathrm{OC}-\mathrm{lhc})\left[n^{\mathrm{HF} x+\mathrm{TPSS} c}\right]$, conv. gauge & 26.6 & 28.5 & 38.2 & 38.8 & 32.0 & 32.0 \\
\hline$(\mathrm{HF} x+\mathrm{OC}-\mathrm{lhc})\left[n^{\mathrm{LSDA}}\right]$, TPSS gauge & 33.4 & 34.4 & 51.1 & 51.1 & 39.3 & 40.0 \\
\hline$(\mathrm{HF} x+\mathrm{OC}-\mathrm{lhc})\left[n^{\mathrm{TPSS}}\right]$, TPSS gauge & 33.5 & 34.6 & 51.5 & 51.5 & 39.6 & 40.3 \\
\hline$(\mathrm{HF} x+$ OC-lhc $)\left[n^{\mathrm{HF} x+\mathrm{TPSS} c}\right]$, TPSS gauge & 26.1 & 27.9 & 36.6 & 37.5 & 29.6 & 31.2 \\
\hline (TPSS $x+$ OC-lhc) $\left[n^{\text {LSDA }}\right]$, TPSS gauge & -14.5 & 15.2 & -29.4 & 29.6 & -19.5 & 20.0 \\
\hline$\left(\right.$ TPSS $x+$ OC-lhc) $\left[n^{\text {TPSS }}\right]$, TPSS gauge & -11.8 & 12.6 & -21.6 & 21.8 & -15.1 & 15.7 \\
\hline$\left(\right.$ TPSS $x+$ OC-lhc) $\left[n^{\text {TPSS }}\right]$, TPSS gauge, red. $\lambda$ & -12.0 & 12.7 & -21.2 & 21.5 & -15.1 & 15.7 \\
\hline
\end{tabular}

optimized-effective potential (OEP) method $^{38}$ or usage of derivatives with respect to the Kohn-Sham orbitals rather than the density (generalized Kohn-Sham method, GKS). ${ }^{2}$ The OEP method is computationally rather challenging. Although still numerically demanding, the GKS scheme is simpler than implementation of the density derivatives by means of the OEP method. The self-consistent implementation of MGGA and HGGA functionals used here follows the GKS scheme. Practical implementations of MGGA and HGGA frequently evaluate them nonself-consistently on LSDA or GGA densi- ties. For comparison purposes we also report below results obtained in this manner.

To implement the OC-lh functionals, we have used a developmental version of the GAUSSIAN $\operatorname{code}^{23}$ that was earlier used to implement the highly constraint-satisfying but empirical local hybrid functional of Ref. 34. The code computes the exact exchange energy density in the conventional gauge, to which we can add a term ${ }^{31}$ that integrates to zero and defines an exchange energy-density gauge that is TPSS -like.

TABLE II. As in Table I, but for barrier heights. Negative mean errors indicate too low barriers. All values are in $\mathrm{kcal} / \mathrm{mol}$.

\begin{tabular}{|c|c|c|c|c|}
\hline & \multicolumn{2}{|c|}{ НТВH38/04 } & \multicolumn{2}{|c|}{ NHTBH38/04 } \\
\hline & ME & MAE & ME & MAE \\
\hline $\mathrm{HF}$ & 12.8 & 13.2 & 8.1 & 9.1 \\
\hline LSDA(SVWN5) & -18.0 & 18.0 & -12.4 & 12.7 \\
\hline PBE & -9.7 & 9.7 & -8.5 & 8.6 \\
\hline TPSS & -8.2 & 8.2 & -9.0 & 9.1 \\
\hline$(\mathrm{HF} x+\operatorname{TPSS} c)\left[n^{\mathrm{LSDA}}\right]$ & 13.0 & 13.3 & 14.6 & 14.6 \\
\hline$(\mathrm{HF} x+\operatorname{TPSS} c)\left[n^{\mathrm{TPSS}}\right]$ & 10.0 & 10.4 & 12.8 & 12.9 \\
\hline HF $x+$ TPSS $c$ & 4.8 & 6.3 & 6.9 & 8.3 \\
\hline$(\mathrm{HF} x+\mathrm{OC}-\mathrm{lhc})\left[n^{\mathrm{TPSS}}\right]$, conv. gauge & 9.2 & 9.8 & 11.8 & 11.9 \\
\hline$(\mathrm{HF} x+\mathrm{OC}-\mathrm{lhc})\left[n^{\mathrm{HF} x+\mathrm{TPSS}}\right]$, conv. gauge & 4.5 & 6.3 & 6.5 & 8.0 \\
\hline$(\mathrm{HF} x+\mathrm{OC}-\mathrm{lhc})\left[n^{\mathrm{LSDA}}\right]$, TPSS gauge & 11.9 & 12.2 & 13.5 & 13.5 \\
\hline$(\mathrm{HF} x+$ OC-lhc $)\left[n^{\mathrm{TPSS}}\right]$, TPSS gauge & 9.0 & 9.6 & 11.7 & 11.9 \\
\hline$\left(\mathrm{HF} x+\right.$ OC-lhc) $\left[n^{\mathrm{HF} x+\mathrm{TPSS} c}\right]$, TPSS gauge & 4.3 & 6.1 & 6.5 & 7.9 \\
\hline$\left(\right.$ TPSS $x+$ OC-lhc) $\left[n^{\mathrm{LSDA}}\right]$, TPSS gauge & -8.5 & 8.4 & -10.0 & $\mathbf{1 0 . 0}$ \\
\hline (TPSS $x+$ OC-lhc) $\left[n^{\text {TPSS }}\right]$, TPSS gauge & -10.0 & 10.0 & -10.0 & 10.1 \\
\hline$\left(\right.$ TPSS $x+$ OC-lhc) $\left[n^{\text {TPSS }}\right]$, TPSS gauge, red. $\lambda$ & -10.4 & 10.4 & -10.5 & 10.5 \\
\hline
\end{tabular}




\section{NUMERICAL TEST FOR ENTHALPIES OF FORMATION AND REACTION BARRIERS}

Tables I and II contain our results for enthalpies of formation and reaction barriers, all obtained on the uncontracted 6$311++\mathrm{G}(3 d f, 3 p d)$ (Refs. 39 and 40) basis set. For all test sets we report the mean error (ME) and the mean absolute error (MAE) from self-consistent Hartree-Fock (HF), LSDA (using the VWN5 parametrization of the correlation energy), ${ }^{41} \mathrm{PBE}-$ GGA, and TPSS-MGGA calculations. These are represented in the first group of four lines in the tables. Against these standard methodologies we compare three classes of beyondGGA functional: exact (HF) exchange added to TPSS correlation, exact (HF) exchange added to the present OC-lh correlation functional (OC-lhc), and approximate (TPSS) exchange added to OC-lh correlation. Within each of these groups, various different implementations and methodologies are included. One of these refers to the level of self-consistency. Where no density argument is given, the corresponding functional has been evaluated self-consistently on its own density. Where an explicit density is specified, the functional has been evaluated post-self-consistently on that density. By changing only the density we can thus assess the importance of proper self-consistency for beyond-GGA functionals.

Another variation refers to the gauge used to make the energy densities unique. In addition to the conventional gauge, in some cases we also report results obtained from the TPSS gauge. ${ }^{31}$ Finally, we also include information on results obtained from a partial reduction of the Lieb-Oxford constant, from the LO to the OC value. This reduction is only partial because it affects the $\lambda$ appearing explicitly in the OC-lh functional, but not the occurrences of $\lambda$ within the component functionals. In Subsections III A and III B we analyze these tables separately for enthalpies of formation and reaction barriers.

\section{A. Enthalpies of formation}

For enthalpies of formation, we use three standard test sets: G3-3 (75 molecules), ${ }^{42}$ G2/97 (148 molecules), ${ }^{43}$ and the superset of both G3/99 (223 molecules, including several large organic molecules) in B3LYP/6-31G(2df, $p)$ geometries. Zero-point energies and finite temperature contributions used a frequency scale factor of $0.9854 .{ }^{44}$ All open-shell species were calculated via spin unrestricted formalisms. The error, which we define as calculated-reference, of the heats of formation is roughly equal and opposite to that of atomization energies. Thus, negative mean errors correspond to overbinding of the molecule. All tested combinations of functionals employing exact (Hartree-Fock) exchange underbind, while all combinations employing approximate (LSDA, GGA, or MGGA) exchange overbind. Of course, these tendencies could be balanced by hybrid functionals mixing exact and approximate exchange, but in the present work hybrids enter merely as a device for implementing HGGA functionals and are not at the focus of investigation.

On these test sets, original self-consistent TPSS is, hands down, the best functional among those included in our tests. This good performance is largely due to error cancellation between approximate TPSS exchange (TPSS $x$ ) and approximate
TPSS correlation (TPSSc), and is thus lost when TPSS $c$ is combined with Hartree-Fock exchange.

As the second group of data of Table I shows, the combination HFx + TPSS $c$ produces significantly worse enthalpies over all three tests sets than does pure TPSS, which is probably related to an insufficient description of static correlation in the used correlation functionals. HGGA correlation functionals (e.g., Ref. 34) can capture some static correlation, but so far only with the help of empirical parameters.

On the other hand, HFx + TPSS $c$ still performs much better than pure HF (and also than LSDA), which shows that TPSS $c$ does recover a substantial amount of dynamic correlation. It makes little difference whether the functional is evaluated post-self-consistently on LSDA or on TPSS densities. A clear improvement is, however, achieved by evaluating it on its own HFx + TPSS $c$ densities, i.e., self-consistently. This improvement shows that self-consistency with regard to the densities is important for fully nonlocal functionals. The common procedure of evaluating complex orbital-dependent functionals on LSDA or GGA densities thus does not permit a proper assessment of their performance.

The third group of data of Table I refers to the combination of $\mathrm{HF}$ exchange with the present OC-lhc. The trend regarding self-consistency is similar to TPSS $c$ : evaluation of the functional on LSDA and on TPSS densities produces very similar results, while HFx + TPSS $c$ densities lead to a significant improvement in performance. Unlike for $\mathrm{HF} x+$ TPSS $c$, however, these are still not the proper self-consistent densities for the HFx + OC-lhc functional, although they should be much closer to those than are LSDA or TPSS densities. Thus, further improvement of the combination HF $x+$ OC-lhc can be expected from a fully self-consistent implementation.

Differences arising from using the conventional gauge instead of the TPSS gauge for the exchange energy density are much smaller than those arising from closer approach to self-consistency, but generally favor the choice of the TPSS gauge. The last group of calculations explores the extent to which OC-lh correlation can benefit from error cancellation with an approximate exchange functional. To allow comparison with the previous sets of calculation we chose TPSS $x$ for this purpose, but we note that error cancellation involving other approximate exchange functional may be larger or smaller. Here, the self-consistent TPSS densities are the closest available approximation to self-consistent TPSS $x+$ OClhc densities. Consistent with our previous observations, the use of these densities produces much better results than that of LSDA densities. Further improvement can be expected from fully self-consistent densities, but even at this level of approximation, the combination TPSS $x+$ OC-lhc is the best performer of all tested functionals, except for fully selfconsistent pure TPSS.

Reduction of $\lambda$ from the LO to the OC value does not produce a consistent behavior, leading to a slight improvement for G3-3 $(0.3-0.5 \mathrm{kcal} / \mathrm{mol})$ and a slight deterioration for $\mathrm{G} 2 / 97$ (0.1-0.2 kcal/mol), while G3/99 was essentially unaffected. This behavior is probably related to the incomplete change of $\lambda$ (in the explicit structure of OC-lhc but not inside the enhancement factor of TPSS) which allows a violation of 
the local Lieb-Oxford condition in the denominator of Eq. (6) for large reduced density gradients.

The behavior of OC-lhc upon consistent reduction of the explicitly appearing $\lambda$, including the PBE enhancement factor within TPSS, is not known yet. We recall, however, that the earlier OC HGGA, ${ }^{22}$ based on total energies [see Eq. (4)], did not have this problem as it employed PW91 GGA in its construction instead of TPSS MGGA. Since PW91 does not feature $\lambda$ in its construction, a reduction of the explicitly appearing $\lambda$ in OC HGGA is already complete, and did, indeed, improve the results. ${ }^{22}$ Based on this experience, it seems possible that a similar improvement will also occur for OC-lh.

The third and fourth groups in Table I assess, from different perspectives, the general performance of the two local mixing functions presented in Eqs. (8) and (9). From the proximity of $(\mathrm{HF} x+\mathrm{OC}-\mathrm{lhc})\left[n^{\mathrm{HF} x+\mathrm{TPSS}}\right]$ results to HF $x+$ TPSS $c$ ones, one can infer that the local mixing parameters of Eq. (8) are quite close to unity, building almost full exact exchange into the local hybrid.

\section{B. Reaction barriers}

For reaction barriers we use two standard test sets. HTBH38/04 (Ref. 45) contains 19 reactions (in total 38 barrier heights) involving hydrogen transfer and NHTBH38/04 (Ref. 46) containing 19 reactions (in total 38 barrier heights) involving non-hydrogen transfer with geometries of QCISD/MG3 level of theory. The negative MEs for TPSS indicate reaction barriers that are too low. All tested combinations of functionals employing exact (Hartree-Fock) exchange overestimate the barriers, while all combinations employing approximate (LSDA, GGA, or MGGA) exchange underestimate them. Once more, these tendencies can be balanced by hybrid functionals mixing exact and approximate exchange, but these are not the aim of the present investigation.

Many of the resulting trends and conclusions are the same as for the case of enthalpies of formation, and we refrain from repeating them here. Instead, we focus on three aspects of the data that are different.

First, we note that combination of approximate TPSS or OC-lh correlation with exact exchange (as long as it is done on self-consistent or nearly self-consistent densities) now produces better results than combination with approximate TPSS exchange. The observation that the use of densities and orbitals obtained from HF exchange lead to significant improvement in reaction barriers, as compared to densities and orbitals obtained from semilocal functionals, extends previous results for standard semilocal functionals $\mathrm{s}^{47}$ to the present class of HGGA-type functionals. Error cancellation between approximate exchange and correlation functionals thus does not play a decisive role for barriers. In this sense, reaction barriers provide a cleaner test ground for the comparison of pure correlation functionals.

Second, for combinations HF $x+$ TPSS $c$ and HF $x+$ OClhc, densities that are closer to self-consistent densities produce better results, just as was the case for enthalpies, but for hydrogen transfer reactions the combination TPSS $x+$ OC-lhc somewhat unexpectedly produces best results on the most ap- proximate (LSDA) densities. This may be due to another type of error cancellation, between densities and functionals, and is probably not an intrinsic feature of the investigated classes of functionals, as it disappears for reactions not involving hydrogen transfer.

Third, the best of all tested functionals (including fully self-consistent TPSS) is now the combination HFx + OC-lhc, evaluated on HFx + TPSS $c$ densities. Although these densities should be close to self-consistent HFx + OC-lhc densities, they are not identical, and further improvement can be expected from a fully self-consistent implementation. Already at the present stage of approximation, however, OC-lh correlation provides the best barrier heights.

Unfortunately, this last result is the opposite of what we found for enthalpies of formation, which again shows that OC-lh HGGA has many of the features like a universally applicable and reliable HGGA should have, but not all of them. A final assessment should await, however, an investigation of the effect of full self-consistency and of consistent reduction of $\lambda$ both of which should further improve OC-lh results. Moreover, the use of other component functionals than TPSS for the OC construction should also be more completely explored.

\section{CONCLUSIONS AND OPEN QUESTIONS}

For enthalpies of formation, OC-lh correlation combined with exact (Hartree-Fock) exchange, as well as TPSS correlation combined with exact exchange, have errors of similar size but opposite sign, neither of them improving on PBE GGA. OC-lh correlation combined with TPSS exchange works better than PBE GGA but does not improve on fully self-consistent TPSS. The fact that the performance of OClh correlation for enthalpies is better in combination with approximate exchange than with exact exchange shows that OClh misses substantial parts of static correlation but benefits significantly from error cancellation between approximate exchange and correlation.

For reaction barriers, the best of all tested functionals is OC-lh correlation combined with Hartree-Fock exchange (HF $x+$ OC-lhc), followed closely by HF $x+$ TPSS $c$. The best results are achieved in both cases on HFx + TPSS $c$ orbitals, and display strong sensitivity to the density and orbitals. The fact that the performance for barriers improves, when combined with exact exchange and evaluated on (nearly) its own orbitals, shows that OC-lh correlation benefits from the reduced self-interaction error arising from exact exchange energies and orbitals, similarly, but to a slightly larger degree, than does TPSS correlation.

For all investigated combinations of MGGA and HGGA functionals and physical properties there are noticeable, and in many cases rather large, changes when LSDA (or GGA) orbitals are replaced by MGGA or HFx + MGGA $c$ orbitals. This flags a warning signal to common post-LSDA and postGGA implementations of complex orbital-dependent functional. Such functionals cannot be reliably assessed in terms of LSDA or GGA orbitals. This finding is consistent with previous similar observations made in the context of other classes of orbital-dependent functionals. ${ }^{47}$ 
Overall, the performance of OC-lh HGGA, as well as that of the earlier OC HGGA, ${ }^{22}$ show that the Lieb-Oxford bound is a potentially useful starting point to construct nonempirical HGGA-type functionals, but also that on its own this bound is not constraining enough to result in a reliable all-purpose functional. Additional constraints are needed. The choices of constraints and of component functional made in the construction of OC HGGA and of OC-lh HGGA are clearly not yet optimal.

Other open questions include the effect of full selfconsistency (with respect to the orbitals or even the densities, using the OEP) and of a consistent reduction of the LiebOxford constant $\lambda$. Previous results ${ }^{22}$ as well as our present analysis suggests that further (probably minor) improvement can be expected along both of these methodological axes, but a definite statement on these matters is still missing.

Finally, we recall that the first HGGAs constructed from the Lieb-Oxford bound ${ }^{22}$ obeyed the rigorous global form of this bound but were not size-consistent, whereas the present OC-lh HGGA is size-consistent but obeys the nonrigorous local form of the bound. LSDA and most nonempirical GGAs (PW91, ${ }^{26} \mathrm{PBE},{ }^{14} \mathrm{PBE}$ as revised by Hammer/Hansen/Nørskov (RPBE) ${ }^{48}$ Vela-Medel-Trickey (VMT) (Ref. 18)) and MGGAs (TPSS, ${ }^{15}$ revTPSS (Ref. 49)) are size-consistent and are constructed to obey Eq. (1) when both $E_{x}$ and $E_{c}$ are approximated, but not necessarily when only $E_{c}$ is approximated. The task to construct sizeconsistent and accurate correlation-only functionals obeying the Lieb-Oxford bound is a major challenge for developers of density functionals. This challenge is not made simpler by the fact that at present only the global Lieb-Oxford bound has been established in full rigour, while the existence of a local version, in a suitable gauge, remains only a plausible conjecture.

\section{ACKNOWLEDGMENTS}

The work at Rice University was supported by the National Science Foundation under Grant Nos. CHE-0807194 and CHE-1110884, the Welch Foundation (C-0036), and the Los Alamos National Labs (Subcontract No. 81277-00110). R.H. thanks the Deutsche Forschungsgemeinschaft (DFG Grant No. HA 57112-1). J.P.P. acknowledges the support of the National Science Foundation through Grant No. DMR0854769 and Cooperative Agreement No. EPS-1003897 with support from the Louisiana Board of Regents. K.C. is supported by grants from FAPESP and CNPq. The authors thank Professor Viktor Staroverov for his implementation of the TPSS gauge of the exact exchange energy density and discussions.

${ }^{1}$ R. M. Dreizler and E. K.U. Gross, Density Functional Theory (SpringerVerlag, 1990).

${ }^{2}$ R. G. Parr and W. Yang, Density Functional Theory of Atoms and Molecules (Oxford University Press, 1989).

${ }^{3}$ W. Kohn, Rev. Mod. Phys. 71, 1253 (1999).
${ }^{4}$ E. H. Lieb, Phys. Lett. A 70, 444 (1979).

${ }^{5}$ E. H. Lieb and S. Oxford, Int. J. Quantum Chem. 19, 427 (1981).

${ }^{6}$ J. P. Perdew, Electronic Structure of Solids (Akademie Verlag, 1991).

${ }^{7}$ M. Levy and J. P. Perdew, Phys. Rev. B 48, 11638 (1993).

${ }^{8}$ W. Kohn and L. J. Sham, Phys. Rev. 140, A1133 (1965).

${ }^{9}$ G. K. L. Chan and N. C. Handy, Phys. Rev. A 59, 3075 (1999).

${ }^{10}$ M. M. Odashima and K. Capelle, J. Chem. Phys. 127, 054106 (2007).

${ }^{11}$ M. M. Odashima and K. Capelle, Int. J. Quantum Chem. 108, 2428 (2008).

${ }^{12}$ E. Räsänen, S. Pittalis, K. Capelle, and C. R. Proetto, Phys. Rev. Lett. 102, 206406 (2009).

${ }^{13}$ E. Räsänen, M. Seidl, and P. Gori-Giorgi, Phys. Rev. B 83, 195111 (2011).

${ }^{14}$ J. P. Perdew, K. Burke, and M. Ernzerhof, Phys. Rev. Lett. 77, 3865 (1996).

${ }^{15}$ J. Tao, J. P. Perdew, V. N. Staroverov, and G. E. Scuseria, Phys. Rev. Lett. 91, 146401 (2003).

${ }^{16}$ M. M. Odashima, K. Capelle, and S. B. Trickey, J. Chem. Theory Comput. 5, 798 (2009).

${ }^{17}$ P. Haas, F. Tran, P. Blaha, L. S. Pedroza, A. J.R. Da Silva, M. M. Odashima, and K. Capelle, Phys. Rev. B 81, 125136 (2010).

${ }^{18}$ A. Vela, V. Medel, and S. B. Trickey, J. Chem. Phys. 130, 244103 (2009).

${ }^{19}$ Y. Zhao and D. G. Truhlar, J. Chem. Phys. 128, 184109 (2008).

${ }^{20}$ J. P. Perdew and K. Schmidt, AIP Conf. Proc. 577, 1 (2001).

${ }^{21}$ J. P. Perdew, A. Ruzsinszky, J. Tao, V. N. Staroverov, G. E. Scuseria, and G. I. Csonka, J. Chem. Phys. 123, 062201 (2005).

${ }^{22}$ M. M. Odashima and K. Capelle, Phys. Rev. A 79, 062515 (2009).

${ }^{23}$ M. J. Frisch, G. W. Trucks, H. B. Schlegel, et al., GAUSsiAN09, Revision G.01, Gaussian, Inc., Wallingford, CT 2009.

${ }^{24}$ P. Dirac, Math. Proc. Cambridge Philos. Soc. 26, 376 (1930).

${ }^{25}$ J. C. Slater, Phys. Rev. B 81, 385 (1951).

${ }^{26}$ J. P. Perdew, J. A. Chevary, S. H. Vosko, K. A. Jackson, M. R. Pederson, D. J. Singh, and C. Fiolhais, Phys. Rev. B 46, 6671 (1992).

${ }^{27}$ C. Lee, W. Yang, and R. G. Parr, Phys. Rev. B 37, 785 (1988).

${ }^{28}$ J. P. Perdew and S. Kurth, Density Functionals for Non-relativistic Coulomb Systems in the New Century, Lecture Notes in Physics, Vol. 620 (Springer, 2003).

${ }^{29}$ F. G. Cruz, K.-C. Lam, and K. Burke, J. Phys. Chem. A 102, 4911 (1998).

${ }^{30}$ P. S. Svendsen and U. von Barth, Phys. Rev. B 54, 17402 (1996).

${ }^{31}$ J. Tao, V. N. Staroverov, G. E. Scuseria, and J. P. Perdew, Phys. Rev. A 77, 012509 (2008).

${ }^{32}$ D. J. Lacks and R. G. Gordon, Phys. Rev. A 47, 4681 (1993).

${ }^{33}$ J. P. Perdew, K. Burke, and M. Ernzerhof, Phys. Rev. Lett. 80, 891 (1998).

${ }^{34}$ J. P. Perdew, V. N. Staroverov, J. Tao, and G. E. Scuseria, Phys. Rev. A 78, 052513 (2008)

${ }^{35}$ P. Mori-Sánchez, A. J. Cohen, and W. Yang, J. Chem. Phys. 124, 091102 (2006).

${ }^{36}$ A. D. Becke, J. Chem. Phys. 122, 064101 (2005).

${ }^{37}$ J. Jaramillo, G. E. Scuseria, and M. Ernzerhof, J. Chem. Phys. 118, 1068 (2003).

${ }^{38}$ T. Grabo, T. Kreibich, and E. K. U. Gross, Mol. Eng. 7, 27 (1997).

${ }^{39}$ A. D. McLean and G. S. Chandler, J. Chem. Phys. 72, 5639 (1980).

${ }^{40}$ R. Krishnan, J. S. Binkley, R. Seeger, and J. A. Pople, J. Chem. Phys. 72, 650 (1980).

${ }^{41}$ S. H. Vosko, L. Wilk, and M. Nusair, Can. J. Phys 58, 1200 (1980),

${ }^{42}$ L. A. Curtiss, K. Raghavachari, P. C. Redfern, and J. A. Pople, J. Chem. Phys. 112, 7374 (2000).

${ }^{43}$ L. A. Curtiss, K. Raghavachari, P. C. Redfern, and J. A. Pople, J. Chem. Phys. 106, 1063 (1997).

${ }^{44}$ L. A. Curtiss, P. C. Redfern, K. Raghavachari, and J. A. Pople, J. Chem. Phys. 114, 108 (2001).

${ }^{45}$ Y. Zhao, B. J. Lynch, and D. G. Truhlar, J. Phys. Chem. A 108, 2715 (2004); 109, 4942 (2006) (erratum).

${ }^{46}$ Y. Zhao, N. Gonzalez-Garcia, and D. G. Truhlar, J. Phys. Chem. A 109, 2012 (2005).

${ }^{47}$ B. G. Janesko and G. E. Scuseria, J. Chem. Phys. 128, 244112 (2008).

${ }^{48}$ B. Hammer, L. Hansen, and J. Nørskov, Phys. Rev. B 59, 7413 (1999).

${ }^{49}$ J. P. Perdew, A. Ruzsinszky, G. I. Csonka, L. A. Constantin, and J. Sun, Phys. Rev. Lett. 103, 026403 (2009). 